\title{
Burrows-Wheeler Transform
}

\author{
Wisely
}




\section{Introduction of BWT}

- Burrows and Wheeler introduced a new compression algorithm based on a reversible transformation now called the Burrows-Wheeler Transform (BWT)

- BWT is applied in data compression techniques such as bzip2 (http://bzip.org/) 


\section{Transform Steps}

(1) Append at the end of a text $T$ a special character $\$$ smaller than any other text character

(2)Form a conceptual matrix $M_{T}$ whose rows are the cyclic shifts of the string $T \$$ sorted in lexicographic order

(3) Construct the transformed text $T^{\text {bwt }}$ by taking the last column of matrix $M_{T}$ 


\section{Example :}

cyclic shifts of the text

\begin{tabular}{|l|l|l|l|l|l|l|l|l|l|l|l|}
\hline m & i & s & s & i & s & s & i & p & p & i & S \\
\hline i & s & s & i & s & s & i & p & p & i & \$ & m \\
\hline s & s & i & s & s & i & p & p & i & S & m & i \\
\hline s & i & s & s & i & p & p & i & S & m & i & s \\
\hline i & s & s & i & p & p & i & S & m & i & s & s \\
\hline s & s & i & p & p & i & S & m & i & s & s & i \\
\hline s & i & p & p & i & S & m & i & s & s & i & s \\
\hline i & p & p & i & S & m & i & s & s & i & s & s \\
\hline p & p & i & S & m & i & s & s & i & s & s & i \\
\hline p & i & S & m & i & s & s & i & s & s & i & p \\
\hline i & S & m & i & s & s & i & s & s & i & p & p \\
\hline S & m & i & s & s & i & s & s & i & p & p & i \\
\hline
\end{tabular}

F

$T^{b w t}$

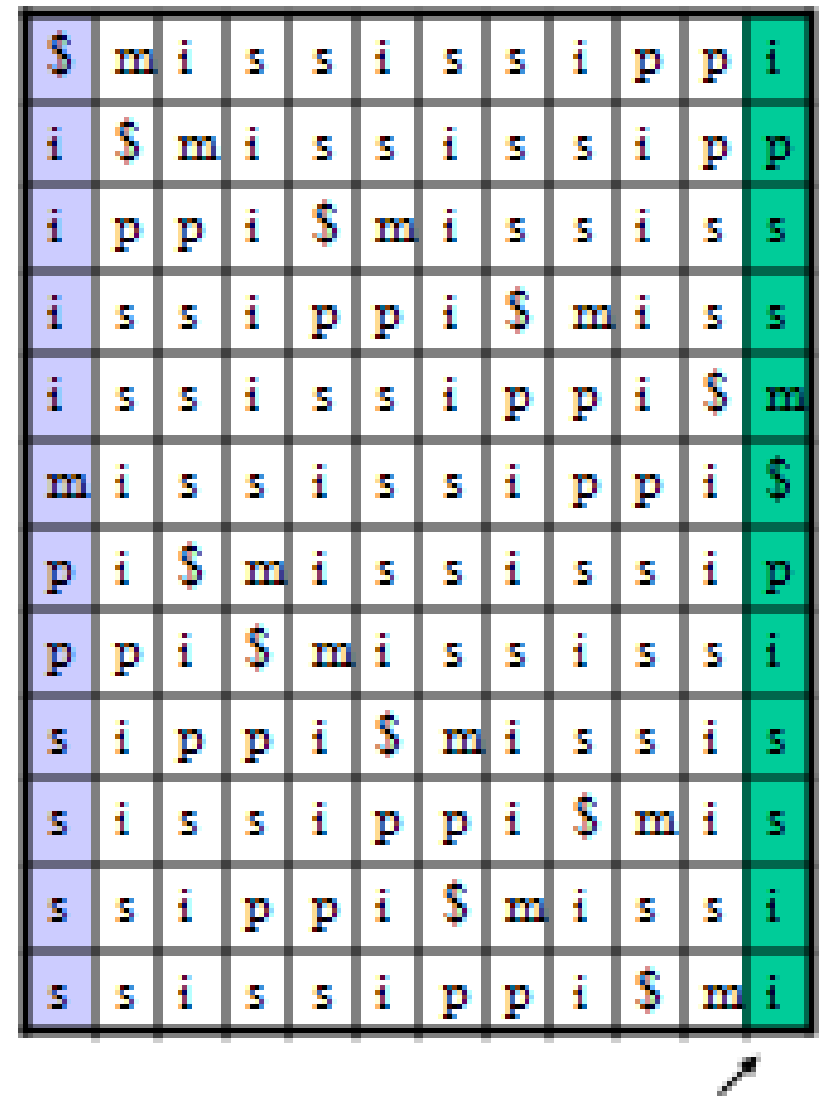

output of BWT

BWT sorts the characters by their context

sorted

lexicographically 


\section{Notation}

(1) Let $C[$ ] be an array of length $|\Sigma|$ such that $C[c]=$ total \# of text characters which are alphabetically smaller than $c$

(2) Let Occ $(c, q)$ denote \# of occurrences of character $c$ in the prefix $T^{b w t}[1, q]$.

(3) Let $L F(i)=C\left[T^{b \omega t}[i]\right]+\operatorname{Occ}\left(T^{b \omega t}[i], i\right)$ 


\section{Example :}

\begin{tabular}{|c|c|c|c|c|c|c|c|c|c|c|c|}
\hline & & & & & & & & & & & \\
\hline$\$$ & & & I & $s$ & & & $s$ & & & & \\
\hline i & $s$ & $\mathrm{~m}$ & $\mathrm{i}$ & 5 & 5 & $\mathrm{i}$ & 5 & $s$ & $i$ & & \\
\hline 1 & $p$ & $\mathrm{p}$ & $i$ & $s$ & $\mathrm{~m}$ & $i$ & 5 & 5 & i & 5 & \\
\hline 1 & $s$ & 5 & $\mathrm{i}$ & $p$ & $\mathrm{p}$ & $\mathrm{i}$ & $s$ & & $\mathrm{i}$ & 5 & \\
\hline$i$ & 5 & 5 & $\mathrm{i}$ & 5 & 5 & $i$ & $p$ & $p$ & $i$ & $\mathbf{S 1}$ & 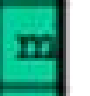 \\
\hline $\mathrm{m}$ & & $s$ & $s$ & $\mathrm{i}$ & 5 & 5 & $\mathrm{i}$ & $p$ & $p$ & i & \\
\hline$p$ & $\mathrm{i}$ & $s$ & $\mathrm{~m}$ & $i$ & 5 & & $\mathrm{i}$ & 5 & 5 & i & \\
\hline$p$ & $p$ & $\mathbf{i}$ & $s$ & & $i$ & 5 & & $i$ & $s$ & 5 & \\
\hline 5 & $i$ & $p$ & $p$ & i & $s$ & & $\mathrm{i}$ & s & $s$ & i & \\
\hline 5 & $\mathrm{i}$ & $s$ & $s$ & $i$ & $\mathrm{p}$ & p & i & s & & & \\
\hline 5 & s & $i$ & $p$ & $p$ & $\mathrm{i}$ & $S$ & & $i$ & $s$ & $s /$ & \\
\hline & & & & & & & & & & $\mathrm{m}$ & \\
\hline
\end{tabular}

C table

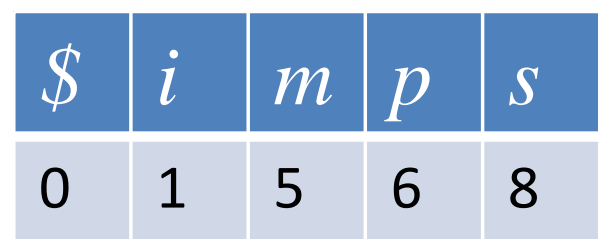

$\operatorname{Occ}(c, q)$

\begin{tabular}{|l|l|l|l|l|}
\hline$\$$ & $i$ & $m$ & $p$ & $s$ \\
\hline 0 & 1 & 0 & 0 & 0 \\
\hline 0 & 1 & 0 & 1 & 0 \\
\hline 0 & 1 & 0 & 1 & 1 \\
\hline 0 & 1 & 0 & 1 & 2 \\
\hline 0 & 1 & 1 & 1 & 2 \\
\hline 1 & 1 & 1 & 1 & 2 \\
\hline 1 & 1 & 1 & 2 & 2 \\
\hline 1 & 2 & 1 & 2 & 2 \\
\hline 1 & 2 & 1 & 2 & 3 \\
\hline 1 & 2 & 1 & 2 & 4 \\
\hline 1 & 3 & 1 & 2 & 4 \\
\hline 1 & 4 & 1 & 2 & 4 \\
\hline
\end{tabular}




\section{Last to Front Mapping}

\begin{tabular}{|c|c|c|c|c|c|c|c|}
\hline & & & & & & & \\
\hline s] & mi & $s$ & 51 & 5 & & & $p$ \\
\hline & $\mathrm{s} \mathrm{m}$ & $\mathrm{i}$ & 5 & $i$ & 5 & 5 & $p$ \\
\hline & \begin{tabular}{l|l}
$p$ & $p$
\end{tabular} & & $S \mid x$ & $x^{i}$ & 5 & $5 \mathrm{i}$ & 5 \\
\hline & s/s & & $p \mid p$ & & s & $\mathrm{mli}$ & 5 \\
\hline 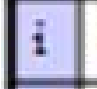 & $5 / 5$ & & 55 & & & $\mathrm{p}$ & $s$ \\
\hline $\mathrm{m}$ & $\mathrm{i} / \mathrm{s}$ & & is & 5 & & $p$ & $\mathrm{I}$ \\
\hline p & $i s$ & $\mathrm{~m}$ & is & & in & 5 & {[} \\
\hline$p$ & 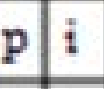 & $\mathrm{s}$ & $\mathrm{mi}$ & 5 & & $i s$ & 5 \\
\hline 5 & $\begin{array}{l}i p \\
\end{array}$ & $p$ & if & $\mathrm{m}$ & 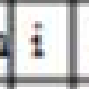 & 5 s & \\
\hline s & $\mathrm{i} / \mathrm{s}$ & 5 & $\mathrm{i}]$ & $p$ & & 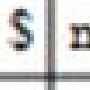 & \\
\hline 1 & si & $p$ & $\mathrm{p}$ & $\mathrm{s}$ & $\mathrm{m}$ & & \\
\hline & & & & & & & \\
\hline
\end{tabular}

- $L F()=$ Last-to-Front Column Mapping

- The character $T^{b w t}[i]$ is located in the first column $F$ at position $L F[i]$

- $L F(10)=C[s]+\operatorname{Occ}(s, 10)=12$.

- Both $T^{b w t}[10]$ and $F[12]$ correspond to the first $s$ in the mississippi 


\section{Backward Search}

- The $L F($ ) mapping allows us to scan the text $T$ backward.

- In other words, we could search a pattern in $T$ backward. (How?) 


\section{Backward Search Algorithm}

Backward_Search( $P[1, p])$

\{

$i=p, c=P[p]$, First $=c[c]+1$, Last $=c[c+1]$;

while ( ( First $\leqq$ Last $)$ and $i \geqq 2$ ) \{

$c=P[i-1]$;

First $=c[c]+\operatorname{Occ}(c$, First-1)+1;

Last $=\mathrm{C}[c]+\operatorname{Occ}(c$, Last $)$;

$i=i-1$;

\}

if ( Last < First ) then return "no occurrence" ;

else return ( First, Last );

\} 


\section{References}

[1] M. Burrows and D. Wheeler (1994).

A Block Sorting Lossless Data Compression Algorithm.

Technical Report 124, Digital Equipment Corporation.

[2] G. Manzini (2001).

An Analysis of the Burrows-Wheeler Transform.

Journal of the ACM, 48(3): pages 407 - 430.

[3] P. Ferragina and G. Manzini (2000).

Opportunistic Data Structures with Applications.

In Proceedings of FOCS, pages 390 - 398.

[4] P. Ferragina, G. Manzini, V. Mäkinen, and G. Navarro (2004).

An Alphabet-Friendly FM-Index.

In Proceedings of SPIRE, pages 150 - 160. 